\title{
Departamento de Informática do SUS - DATASUS: a gestão de dados de saúde no Brasil e sua contribuição para a in- clusão digital
}

\author{
Dayanne da Silva Prudencio \\ Universidade Federal do Estado do Rio de Janeiro, Brasil \\ Carlos Alberto Ferreira \\ Universidade Federal do Estado do Rio de Janeiro, Brasil
}

ORIGINAL

\begin{abstract}
Resumo
Objetivo. Apresenta o Departamento de Informática do Sistema Único de Saúde (DATASUS), um órgão público brasileiro subordinado à Fundação Nacional de Saúde (Funasa). O órgão recebe e armazena dados produzidos pelo ministério e pelas secretarias estaduais de saúde de todo país. Além disso, assessora secretarias municipais de saúde no estabelecimento de sistemas de informação e incorporação de tecnologias que permitam mais eficiência na gestão de dados e informações de caráter estratégico.

Método. Pesquisa é exploratória-descritiva, utiliza procedimentos bibliográficos e documentais para coleta dos dados. Utilizou-se abordagem quantitativa e qualitativa para análise dos dados e demonstração dos resultados. Como técnica de análise de dados adota-se a análise de conteúdo segundo Bardin (2011). Os documentos analisados foram recuperados no sítio oficial do DATASUS, já a bibliografia examinada foi obtida a partir de pesquisa no idioma português, espanhol e inglês na LISA e BRAPCI.

Resultados. Conclui que o DATASUS é um órgão de importância e que oferece importantes contribuições para a criação de uma infraestrutura tecnológica e informacional de dados e informações em saúde no Brasil. Ainda se verificou que o órgão tem prestado serviços relevantes para sanear a desigualdade e inclusão digital que ainda existem no país. Sugere-se que bibliotecários brasileiros se engajem em atividades de promoção a competência informacional em saúde e para tanto recomenda apoio nas boas práticas, modelos e infraestrura existentes no sistema de informação.
\end{abstract}

Palavras-chave:

Departamento de Informática do Sistema Único de Saúde - DATASUS; Gestão de dados; Governança de dados; Sistema de informação

\section{SUS Computer Department - DATASUS: health data management in Brazil and its contribution to digital inclusion}

\begin{abstract}
Objective. It introduces the Department of Informatics of the Unified Health System (DATASUS), a Brazilian public agency subordinated to the National Health Foundation (Funasa). The agency receives and stores data produced by the ministry and by state health departments throughout the country. In addition, it advises municipal health secretariats on the establishment of information systems and the incorporation of technologies that enable more efficiency in the management of data and information of a strategic nature.

Method. The research is exploratory-descriptive, using bibliographic and documentary procedures for data collection. A quantitative and qualitative approach was used for data analysis and demonstration of results. As a technique for data analysis we adopted the content analysis according to Bardin (2011). The documents analyzed were retrieved from the official DATASUS website, while the bibliography examined was obtained from research in Portuguese, Spanish and English in LISA and BRAPCI. Results. Concludes that DATASUS is an important body that offers important contributions to the creation of a technological and informational infrastructure of data and health information in Brazil. It was also found that the agency has provided relevant services to remedy the inequality and digital inclusion that still exist in the country. It is suggested that Brazilian librarians engage in activities to promote health information competency and therefore recommends support for existing good practices, models and infrastructure in the information system.
\end{abstract}

Keywords:

Departamento de Informática do Sistema Único de Saúde - DATASUS; Data management; Data governance; Information system 


\section{Introdução}

Criado em 1991, o Departamento de Informática do Sistema Único de Saúde (DATASUS) é um órgão público brasileiro subordinado à Fundação Nacional de Saúde (Funasa). Sua principal responsabilidade é prover os órgãos do Sistema Único de Saúde (SUS) com infraestrutura de sistemas de informação e suporte de informática visando contribuir no processo de planejamento, operação e controle.

Trata-se da maior e principal fonte de armazenamento de dados da população brasileira e também de todo o Ministério da Saúde. O órgão recebe e armazena dados produzidos pelo ministério e pelas secretarias estaduais de saúde de todo país. Além disso, assessora secretarias municipais de saúde no estabelecimento de sistemas de informação e incorporação de tecnologias que permitam mais eficiência na gestão de dados e informações de caráter estratégico.

Atualmente o repositório de dados incorpora dados de 11 macroestruturas informacionais, a saber: Cadastros Nacionais, Epidemiológicos, Ambulatoriais, Regulação, Sociais, Financeiro, Gestão, Hospitalares, Estruturantes, Eventos Sociais e Sisconferência. Cada uma destas macroestruturas divide-se em pelo menos duas outras infraestruturas de dados.

Na mesma linha, é importante informar que o DATASUS disponibiliza informações e padrões de interoperabilidade em saúde indicando as premissas, políticas e especificações técnicas que subsidiam o intercâmbio de informações entre os sistemas de saúde dos órgãos federais, municipais e estaduais. Desta forma, pode-se falar de uma infraestrura nacional e interoperável de dados, estabelecendo indicadores que contribuam efetivamente na tomada de decisão e de construção de políticas públicas.

Outrossim, pode-se conceber o DATASUS como o mais importante repositório de dados de saúde em esfera nacional e uma relevante fonte de informação para as práticas informacionais realizadas para os profissionais de informação brasileiros. Contudo, essa infraestrutura tem sido pouco debatida e analisada na produção científica biblioteconômica e de Ciência da Informação, vide dados apresentados na seção 04. Sendo assim, a pesquisa em tela defende maior promoção e uso do repositório na divulgação de para disseminação de informação em saúde.

Neste contexto, este trabalho objetiva explorar as potencialidades do DATASUS como infraestrutura informacional e recurso para o fomento de pesquisas interdisciplinares entre os domínios da Saúde, Tecnologia e Ciência da Informação.

\section{Metodologia}

A pesquisa se caracteriza como sendo exploratória-descritiva, no que se refere ao seu objetivo, e desenvolve procedimentos bibliográficos e documentais para coleta dos dados. Além disso, utiliza-se abordagem quantitativa e qualitativa para análise dos dados e demonstração dos resultados. Para analisar os dados coletados, adotou-se a técnica de análise de conteúdo segundo diretrizes de Bardin (2011).

Os documentos analisados foram recuperados no sítio oficial do DATASUS, a bibliografia examinada foi obtida a partir de pesquisa bibliográfica no idioma português, inglês e espanhol nas seguintes fontes: Base de Dados em Ciência da Informação (BRAPCI) e na Library, Information Science \& Technology Abstract (LISA).

Para a consecução da segunda fase que trata da exploração do material, definida por Bardin como "a fase longa e fastidiosa [da pesquisa, pois] consiste essencialmente em operações de codificação, decomposição ou enumeração, em função de regras previamente formuladas" (BARDIN, 2011, p.131), fez-se uma leitura completa dos artigos selecionados anteriormente e analisou-se segundo as seguintes categorias: título, periódico, dados de autoria e ênfase temática.

\section{Revisão de literatura}

Moraes e Gómez (2007, p. 558) designam informação em saúde como "o enquadramento dos significados da saúde reconstruídos e alargados na nova ordem da medicalização e das instituições - primeiro de atendimento e depois de ciência e tecnologia em saúde".

Já Moraes e Santos (1998, p. 39) compreendem informação em saúde como um campo temático que se impõe, inter-relacionando níveis de investigação, de saberes, de modos de caminhar e olhar o mundo. Nesta linha, é 
factível compreender que as tecnologias da informação e comunicação tem contribuído para o desenvolvimento de uma função macro da informação em saúde.

Nesta linha, a tecnologia tem sido entendida como um recurso fundamental para promover a democratização da saúde, aprimoramento dos processos de trabalho em saúde e melhoria de sua gestão. Ou seja, opera tanto como ferramenta para desenvolvimento dos sistemas de informação quanto como canal para disponibilização das informações úteis a sociedade.

Outrossim, observa-se um alinhamento entre tecnologia, subsídios a políticas de saúde e reformas do sistema de saúde (Galian e Louzano, 2014). Isto é, desde a década de 70 quando foram iniciados os movimentos relacionados às reformas do sistema de saúde no Brasil e se intensificaram as pesquisas e comunicações sobre os estudos de epidemiologia, ocorreu uma necessidade de ampliar a comunicação das descobertas para as autoridades e orientar a população sobre as questões de saúde (MORENO; COELI; MUNCK, 2009). Neste sentido, estas infraestruturas info-comunicacionais são entendidas como integradas em todo fluxo informacional, ou seja, desde a produção da informação até sua disseminação.

Paralelamente a este contexto, ocorrem outros movimentos de pesquisadores de várias áreas do conhecimento (Medicina, Informática, Ciências Sociais entre outros) pró-desenvolvimento e aplicação da tecnologia da informação e comunicação (TIC) no âmbito do sistema de saúde brasileiro. Tais iniciativas culminam com o advento do termo informática em saúde ou informática médica. O campo dá seus primeiros passos no Brasil em 1975, no interior do Núcleo de Tecnologia de Educação em Saúde na Universidade Federal do Rio de Janeiro (UFRJ), mas é em 1983 que é normatizado com a criação de novos grupos especificamente dedicados a esta área de pesquisa e ensino (SABBATINI, 1998).

Em uma tessitura de ocorrências e apelo à promoção da saúde e disseminação de informações sobre saúde como direito ocorre em 1986 a $8^{\text {a }}$ Conferência Nacional de saúde, que teve três grandes eixos: "Saúde como direito e cidadania", "Reformulação do Sistema Nacional de Saúde" e "Financiamento Setorial". Essa é assumida como um marco histórico na trajetória da política pública de saúde brasileira. (BRAVO, 2009)

Essa conferência teve significativos resultados, ressalta-se na pesquisa em tela, as alterações relacionadas a abordagem do tema na Constituição e, posteriormente, a criação da Lei 8.080 de 19 de setembro de 1990, que dispõe sobre as condições para a promoção, proteção e recuperação da saúde, a organização e o funcionamento dos serviços correspondentes e dá outras providências (BRASIL, 1990).

Da legislação citada, importa-nos especificamente o artigo 7 pois estabelece o direito de informação, às pessoas assistidas, sobre a própria saúde. Portanto, evidencia-se, do ponto de vista normativo, a relação dos fenômenos informacionais com o sistema de saúde. Esse e outros inúmeros fatores contribuem para que o campo de informação em saúde desenvolva-se empiricamente e teoricamente tanto como campo disciplinar de ensino, quanto como de pesquisa.

Lembremo-nos que informação em saúde é um dos itens anunciados para mensuração do Índice de Desenvolvimento Humano (IDH), sendo fundamental ao processo de tomada de decisões e construção de indicadores que orientam as ações e estratégias fixadas nas políticas de saúde (TARGINO, 2009).

Coadunam, neste contexto, a necessidade de repensar o sistema de saúde de maneira integrada, com foco em boas práticas de gestão, diagnóstico e terapêutica. Integração permitida em grande parte graças às perspectivas interdisciplinares que se estabelecem no campo do trabalho e que fecundam e abrem espaço para trocas e combinações de múltiplos saberes e o desenvolvimento de grandes infraestruturas informacionais.

Como reflexo da reforma sanitária, manifesta-se uma demanda de informações em saúde atualizadas e detalhadas, conforme veremos na subseção a seguir.

\subsection{Demandas de publicização de dados sobre informação e saúde: respostas do estado brasileiro}

A primeira resposta do Estado a este pleito ocorre em 1977, com a criação do Sistema Nacional de Registros de óbitos no Brasil, uma iniciativa do Instituto Nacional de Assistência Médica da Previdência Social (INAMPS), que, logo após, nos anos 80, desenvolveu dispositivos de planejamento e controle da produção hospitalar. Lima et al (2009) alertam que estas são as primeiras iniciativas de disseminação de informações em saúde, ainda limitadas ao público interno da área da previdência social. 
O primeiro grande avanço orientado às aplicações para apoiar as demais ações da área de Saúde são da década de 90, com a criação do DATASUS. De acordo com Lima et al (2009), desde sua criação, o órgão tem como prática e missão a disseminação de informações em saúde de forma segura, pública e gratuita, bem como, de instrumentos que facilitam o seu uso.

O DATASUS disponibiliza aos cidadãos e gestores de dados em saúde um conjunto de 5 metodologias: desenvolvimento de software, gerenciamento de projeto, desenvolvimento de serviços, administração de dados e gerenciamento de processos; 4 diretrizes: Arquitetural, Guia de Desenvolvimento, Arquitetural BackEnd, Arquitetural FrontEnd e 01 Processo de Gerenciamento e Desenvolvimento Mobile. Esta orientação de compartilhamento informacional, alinha-se com as melhores práticas de Sistemas de Gestão e Qualidade que visam a interação de processos e recursos necessários a agregar valor e realizar resultados para as partes interessadas pertinentes e enquadram-se no requisito de infraestrutura da ISO 9001 - norma que certifica um sistema de gestão da qualidade.

Mediante o regime de padronização metodológica conferida pelas infraestruturas do DATASUS é possível que os gestores informacionais de dados e informações em saúde possam executar o seu trabalho de uma maneira mais segura, fácil e confiável, haja vista que um operador de garantia de qualidade já executou testes de conformidade. Neste sentido, as padronizações envolvem padrões técnicos (especificações) bem como os procedimentos operacionais para produção dos sistemas.

Observa-se ainda, que estas metodologias orientam processos de combinação do conhecimento (Nonaka, 1991). Ou seja, a gerência do DATASUS ao compartilhar seus conhecimentos explícitos sobre metodologias e boas práticas em gerência de projetos, permite que outras instituições de saúde ao identificar oportunidades de negócio em algum processo, na estrutura, sistema ou produto de saúde saiba mensurar sua viabilidade e o desenvolva com efetividade. Ou seja, poderão conduzir projetos de forma planejada e científica, desenvolvendo assim, novos conhecimentos explícitos. (Brasil, 2001).

Ancorado no princípio de suporte a decisão baseada em evidência, merece destaque o desenvolvimento, gestão e manutenção de sistemas de informação como o SisCam - Sistema de Informação do Câncer da Mulher que presta relevantes serviços na sistematização a obtenção de dados, que permitem avaliar e monitorar a evolução e o desfecho do câncer do colo do útero no Brasil, bem como, o GAL - Gerenciador de Ambientes Laboratoriais que informatiza e realiza ações de melhoria no Sistema Nacional de Laboratórios de Saúde Pública das Redes Nacionais de Laboratórios de Vigilância Epidemiológica e Vigilância em Saúde Ambiental. A rápida e eficiente disponibilização de dados do órgão permitiu monitorar a mortandade de macacos com febre amarela silvestre em áreas urbanas ocorrida em 2017 e assim desenvolver o planejamento de campanhas de vacinação de acordo com níveis de criticidade.

No que tange à arquitetura de informação, o repositório disponibiliza orientações em 3 seções: Padrões e Normas, Catálogo de Serviços e Termos de Cessão. Esse conjunto de diretrizes permite que os provedores de dados e os provedores de serviços conheçam a arquitetura prevista na representação de conceitos e, portanto, desenvolvam infraestrutura segura, confiável e que permita um eficiente compartilhamento e cooperação de dados em saúde.

Em consonância com as diretrizes da lei n 12.527/2011 - popularmente conhecida como LAl, e, que regulamenta o direito constitucional de acesso às informações públicas, o repositório disponibiliza distintas infraestruturas de acesso à informação, tais como: Portal de Saúde do Cidadão, Cartão Nacional do SUS, Informações de saúde (TABNET), Informações Financeiras, Serviços, Publicações e Sistemas de Gestão. Tais dados contribuem para "subsidiar análises objetivas da situação sanitária, tomadas de decisão baseadas em evidências e elaboração de programas de ações de saúde" (DATASUS, 2018).

É importante citar que estas infraestruturas não invalidam o Sistema eletrônico do Serviço de Informações ao Cidadão (e-SIC), portanto, qualquer cidadão civil pode abrir uma consulta endereçada ao DATASUS com seus questionamentos e dúvidas.

As consultas acerca de informações e saúde são relevantes para promover a nossa própria saúde, a saúde de outrem, para apoiar as melhores decisões, evitar riscos para a saúde, detectar e diagnosticar problemas de saúde e buscar os melhores serviços de saúde disponíveis. Contudo, sabe-se que apenas uma parte da população possui competência informacional suficientes para desenvolver buscar, acessar e compreender as informações de saúde relevantes às suas necessidades informacionais. 


\begin{abstract}
A competência em informação vai além da busca pela informação, uma vez que considera processos intelectuais superiores como interpretação, avaliação, organização da informação e seu uso, com vistas à interiorização de conhecimentos, habilidades e valores que levem ao aprendizado independente, auto orientado, ao longo da vida. (DUDZIAK, 2001, p. 59-60).
\end{abstract}

Neste sentido, relaciona-se a divulgação de dados e informações em/de saúde com o acesso e compreensão destas informações. Contudo, muitas vezes observa-se uma lacuna, é neste que emergem os estudos de literacia informacional em saúde.

Parker e Kreps (2005, tradução nossa) concebem literacia informacional em saúde como à capacidade de obter, processar e compreender a informação e os serviços necessários para tomar decisões em relação à saúde básica. Os autores acrescentam que essas habilidades devem permitir que o usuário possa obter melhores cuidados e mais qualidade em suas demandas.

Não bastante, Parker e Kreps (2005, tradução nossa) afirmam que os princípios da literacia permitem redução dos custos do sistema de saúde pois evitam consultas e procedimentos desnecessários, melhoria no atendimento e maior eficiência e alcance das políticas públicas de saúde.

A literacia informacional em saúde relaciona-se enquanto prática com outras ações, tais como os programas educacionais de alfabetização em saúde, os programas de design da informação, programas de treinamentos de comunicação de informação em saúde (Parker e Kreps, 2005; Fineberg, 2004) e acrescentamos os programas de inclusão digital.

Na pesquisa que ora desenvolvemos, importa-nos este último pois o DATASUS desenvolve ações relacionadas à inclusão digital, tais como: o Curso de Introdução à Informática Básica, que faz parte do Programa de Inclusão Digital (PID) dos conselhos de saúde municipais; o desenvolvimento do Cartão SUS Digital, que permite que através de um aplicativo de celular o cidadão informe alguns dados e obtenha a primeira ou segunda via do cartão. A medida propicia economia com os gastos de emissão impressa, unifica as informações do paciente e evita fraudes. Segundo Moraes et al (2009, p. 880) a inclusão digital em saúde pressupõe a inclusão informacional em saúde e que se manifesta em:

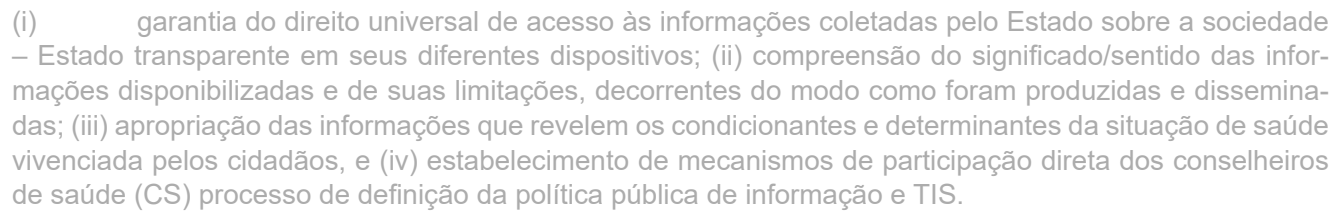

Não obstante, a inclusão informacional em saúde capacita para que o usuário, que também é produtor de dados e de informação em saúde, tome decisões mais conscientes acerca da divulgação de seus dados e levanta questões éticas importantes sobre os méritos e modelo de infraestrutura informacional que serve toda a população. Por exemplo, dados de saúde acerca da população moradora de rua e/ou em vulnerabilidade social, como são tratados, recuperados e acessados? Essas e outras questões são para pesquisas futuras.

Em outra linha, a literacia informacional em saúde alinhada à inclusão informacional em saúde atua sobre o nível de eficiência das iniciativas do Estado, como, por exemplo, serviços oferecidos pelo DATASUS. Ou seja, a população utiliza mais e melhor os serviços digitais de saúde, há redução do uso inadequado dos serviços de saúde e melhora-se a experiência e satisfação do utente do sistema de saúde.

Discutir sobre a inclusão informacional em saúde também nos suscita uma reflexão acerca do papel dos bibliotecários e das bibliotecas neste contexto, isso porque entendemos que estes podem atuar em distintas frentes, como, por exemplo, em programas de competência informacional, com vistas a contribuir para que a população melhore suas práticas informacionais.

Sendo assim, entendemos que os bibliotecários brasileiros precisam engajar-se mais em atividades de inclusão informacional em saúde. Alguns caminhos que nossa pesquisa indica são: atividades de intraempreendorismo, participação em projetos de extensão universitária e parcerias com órgãos do terceiro setor.

De acordo com Lima et al (2009), o sítio do DATASUS é consultado principalmente por profissionais (servidores) das secretarias municipais de saúde, e por aqueles vinculados às secretarias estaduais e às universidades. Ou 
seja, releva-se uma interface técnico-científica que vem fortalecendo o SUS e o desenvolvimento de políticas públicas de saúde.

Desde sua criação, o DATASUS já desenvolveu mais de 200 sistemas de informação, prestou mais de 1000 de assessorias a secretarias estaduais e municipais de saúde e consolida-se como o principal provedor de soluções em tecnologia da informação na área de saúde.

A adoção e ampliação de instrumentos como o DATASUS também contribuem para diminuir a desigual inclusão digital que afeta cidadãos civis, gestores, prestadores de serviços em saúde e trabalhadores do setor.

Nesta linha, ações de intraempreendorismo, podem ser vinculadas como atividade de trabalho e pesquisa em Startups focadas em desenvolvimento de soluções para a área de saúde como forma de auxiliar o sistema de saúde de maneira plena, isto é, os gestores, os médicos e os pacientes em suas necessidades informacionais

\section{Resultados}

Ao buscarmos explorar os aportes e relacionamentos entre a produção científica brasileira da área de Biblioteconomia e Ciência da Informação sobre o DATASUS, ou que o utilize enquanto campo empírico, realizamos pesquisa em duas diferentes fontes: os periódicos brasileiros de Biblioteconomia e Ciência da Informação indexados na base BRAPCI e LISA.

Cumpre informar que a BRAPCl é a principal fonte nacional, haja vista que mantém indexados 57 títulos de periódicos da área de Ciência da Informação e sua cobertura temporal cobre do ano de 1972 em diante. Já a LISA é uma das principais fontes internacionais da área, indexando importantes periódicos e anais de congressos.

Optou-se por utilizar o mesmo protocolo de pesquisa em ambas as fontes, ou seja, as buscas foram realizadas pelos termos: "DATASUS" e "Departamento de informática do Sistema Único de Saúde" nos campos, título e palavras chaves. Não ocorreu restrição de idioma e tampouco temporal. Os dado são apresentados na tabela a seguir:

Tabela 01 - Processo de recuperação da informação

\begin{tabular}{|l|l|c|}
\hline \multicolumn{2}{|c|}{ BASE } & \multicolumn{1}{|c|}{ FILTRO } \\
\hline LISA & Título and palavras chaves & 1 \\
\hline & Título and palavras chaves & 1 \\
BRAPCI & & \\
\hline
\end{tabular}

Fonte: elaborado pelos autores (2019).

Segundo o caminho metodológico traçado, iniciou-se a análise dos 2 artigos recuperados e mediante as categorias criadas e seus respectivos indicadores, temos:

Tabela 01 - Processo de recuperação da informação

\begin{tabular}{|c|c|c|c|}
\hline Título & Peródico & Autoria & Ênfase temática \\
\hline $\begin{array}{l}\text { Qualidade de dados dos } \\
\text { sistemas de informação } \\
\text { do Datasus: análise críti- } \\
\text { ca da literatura }\end{array}$ & $\begin{array}{l}\text { Ciência da Informação } \\
\text { em Revista, n. 3, v. 5, p. } \\
\text { 13-19, } 2018\end{array}$ & $\begin{array}{l}\text { PICCOLO, Daiane Mar- } \\
\text { cela }\end{array}$ & $\begin{array}{l}\text { Garantia da qualidade } \\
\text { dos dados dispostos no } \\
\text { DATASUS }\end{array}$ \\
\hline $\begin{array}{l}\text { Comportamiento in- } \\
\text { formacional y políticas } \\
\text { públicas de información: } \\
\text { consideraciones teóricas } \\
\text { alrededor del caso de } \\
\text { DATASUS en Brasil }\end{array}$ & $\begin{array}{l}\text { Investigación Bibliotecoló- } \\
\text { gica. May-Aug. } 2016 \text {, vol. } \\
30, \text { n. } 69 \text {, p.19-42 }\end{array}$ & $\begin{array}{l}\text { Berrío-Zapata, Cristian } \\
\text { Assis-Rodrigues, Fernan- } \\
\text { do de } \\
\text { Cassiano-Lopes, Rita de } \\
\text { Cássia } \\
\text { Grossi de Carvalho, An- } \\
\text { gela Maria } \\
\text { Gonçalves-Santana, } \\
\text { Ricardo Cesar }\end{array}$ & $\begin{array}{l}\text { Modelos e teorias de } \\
\text { comportamento informa- } \\
\text { cional e sua influência na } \\
\text { formulação de políticas } \\
\text { de informação }\end{array}$ \\
\hline
\end{tabular}

Fonte: elaborado pelos autores (2019). 
A análise dos artigos recuperados, indicam uma maior orientação à estudos que analisam as políticas de informação em saúde e o papel do DATASUS neste contexto. Neste sentido, analisasse a qualidade destes dados e como efetivamente eles podem contribuir e manifestar a política de informação e regime de informação vigente.

No que tange a filiação destes autores observa-se predomínio de doutores em Ciência da Informação, porém com graduações interdisciplinares a área de informação

\section{Quadro 02 - Análise de autoria}

\begin{tabular}{|l|l|}
\hline \multicolumn{1}{|c|}{ Autoria } & \multicolumn{1}{|c|}{ Formação } \\
\hline PICCOLO, Daiane Marcela & $\begin{array}{l}\text { Mestrado em Ciência da Computação } \\
\text { Graduação em Tecnologia em Processamento de Dados }\end{array}$ \\
\hline Berrío-Zapata, Cristian & $\begin{array}{l}\text { Doutorado em Ciência da Informação } \\
\text { Graduação em Psicologia }\end{array}$ \\
\hline Assis-Rodrigues, Fernando de & $\begin{array}{l}\text { Doutorado em Ciência da Informação } \\
\text { Graduação em Sistemas de Informação }\end{array}$ \\
\hline Cassiano-Lopes, Rita de Cássia & $\begin{array}{l}\text { Mestrado em Ciência da Informação } \\
\text { Graduação em Tecnologia em Processamento de Dados }\end{array}$ \\
\hline Grossi de Carvalho, Angela Maria & $\begin{array}{l}\text { Doutorado em Ciência da Informação } \\
\text { Graduação em Comunicação Social - habilitação em Jornalismo }\end{array}$ \\
\hline Gonçalves-Santana, Ricardo Cesar & $\begin{array}{l}\text { Doutorado em Ciência da Informação } \\
\text { Graduação em Pedagogia }\end{array}$ \\
\hline
\end{tabular}

Fonte: elaborado pelos autores (2019).

Todos os autores atuam como docentes em universidades públicas brasileiras. Observa-se predominância de 4 professores da região sudeste, um professor da região norte e um da região sul.

Embora o DATASUS seja uma importante infraestrutura informacional brasileira, este tem sido pouco debatido e analisado na produção científica do espectro informacional. Consideramos que estudos relacionados à padrões de metadados, mecanismos de preservação digital, gestão da informação, competência informacional entre outros possam ser desenvolvidos tendo o DATASUS como campo teórico-empírico.

Pode-se inferir que a ampliação destes resultados contribua para uma melhor gestão de dados em saúde, ampliação do papel do profissional da informação como gestor de dados e não menos para o reconhecimento da área de Ciência da Informação e Biblioteconomia como produtora de conhecimento.

\section{Conclusões}

O objetivo principal do estudo foi apresentar a importância do DATASUS e suas contribuições para a criação de uma infraestrutura tecnológica e informacional de dados e informações em saúde no Brasil. Ainda verificou que o órgão tem prestado serviços relevantes para sanear a desigualdade e inclusão digital que ainda padece o país.

Sugere que bibliotecários brasileiros se engajem em atividades de promoção a competência informacional em saúde e para tanto recomenda apoio nas boas práticas, modelos e infraestrura existentes no DATASUS.

Acredita-se, ainda, que é possível que a produção científica e empírica sobre a infraestrutura permita que o Ministério da Saúde amplie seu alcance e perspectiva informacional, de modo a tornar as políticas de dados e disseminação de informação em saúde mais eficiente e efetiva frente as necessidades da sociedade. 


\section{Referências}

BARDIN, Laurence. Análise de conteúdo. São Paulo: Edições 70, 2011. 279 p.

BRASIL. Ministério da saúde. Metodologia de gerência de projetos do DATASUS. Brasília: Ministério da Saúde, 2001. Disponível em: http://bvsms.saude.gov.br/bvs/publicacoes/metodologia datasus.pdf. Acesso em: 20 mar. 2019.

DUDZIAK, Elisabeth Adriana. A information literacy e o papel educacional das bibliotecas. 2001. Dissertação (Mestrado) - Programa de Pós-Graduação em Ciências da Comunicação, Escola de Comunicações eArtes, Universidade de São Paulo (USP), São Paulo, 2001. Disponível em:< http://www.teses.usp.br/teses/disponiveis/27/27143/ tde-30112004-151029/pt-br.php>. Acesso em: 20 jan. 2019.

LIMA, Claudia Risso de Araujo et al. Departamento de Informática do SUS - DATASUS A Experiência de Disseminação de Informações em Saúde. In. Ministério da Saúde. A experiência brasileira em sistemas de informação em saúde. Brasília: Editora do Ministério da Saúde, 2009.

MORAES, Ilara Hämmerli Sozzi de, \& Gómez, Maria Nélida González de. (2007). Informação e informática em saúde: caleidoscópio contemporâneo da saúde. Ciência \& Saúde Coletiva, v.12, n.3, p. 553-565. Disponível em: http://www.scielo.br/scielo.php?script=sci arttext\&pid=S1413-81232007000300002. Acesso em: 20 ago. 2018.

MORAES, Ilara Hämmerli Sozzi de; Santos, Silvia R. Fontoura Rangel dos. Informação em Saúde: Os Desafios Continuam. Ciência \& Saúde Coletiva, v.3, n.1, p. 37-51, 1998. https://dx.doi.org/10.1590/1413-812319983102772014. Acesso em: 20 set. 2018.

MORAES, Ilara Hämmerli Sozzi de et al. Inclusão digital e conselheiros de saúde: uma política para a redução da desigualdade social no Brasil. Ciência \& Saúde Coletiva, v.14, n. 3, p.879-888, 2009. Disponível em: https://dx.doi. org/10.1590/S1413-81232009000300023. Acesso em: 05 jul. 2019.

TARGINO, Maria das Graças. Informação em Saúde: potencialidades e limitações. Informação \& Informação, [S.I.], v. 14, n. 1, p. 52-81, jul. 2009. Disponível em: http://www.uel.br/revistas/uel/index.php/informacao/article/ view/1845/2891. Acesso em: 29 mar. 2019. 


\section{$\longrightarrow$ Departamento de Informática do SUS - DATASUS: a gestão de dados de saúde no Brasil e sua contribuição para a inclusão digital}

\section{Dados dos autores}

Dayanne da Silva Prudencio

Professora do Departamento de Biblioteconomia da Universidade Federal do Estado do Rio de Janeiro (UNIRIO). Doutoranda em Ciência da Informação pelo convênio IBICT/UFRJ.

dayanneprudencio@gmail.com

\section{Carlos Alberto Ferreira}

Doutor em Ciência da Informação pela Universidade Federal do Rio de Janeiro em convênio com o Instituto Brasileiro de Informação em Ciência e Tecnologia, Professor Adjunto do Curso de Biblioteconomia da Universidade Federal do Estado do Rio de Janeiro (UNIRIO).

carlos.cafe@unirio.br

Received: 2019-10-28

Accepted: 2021-01-09

\section{$(\mathrm{cc}) \mathbf{E Y}$}

This work is licensed under a Creative Commons Attribution 4.0 United States License.

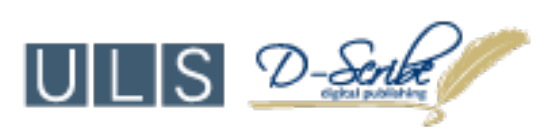

This journal is published by the University Library System of the University of Pittsburgh as part of its D-Scribe Digital Publishing Program and is cosponsored by the University of Pittsburgh Press 